\title{
Unusual Case Presentation of Intermittent Aortic Prosthetic Valve Dysfunction
}

\author{
Hakim Irfan Showkat ${ }^{*}$, Srivastava $\mathbf{R}^{1}$, Mishra $\mathbf{R}^{1}$, Sharma $\mathbf{V}^{1}$, \\ Krishna $\mathrm{CK}^{1}$, Asotra $\mathrm{S}^{1}$, Ahlawat $\mathrm{V}^{2}$, Yadava $\mathbf{O P}^{2}$, Anwar $\mathrm{S}^{1}$ and \\ Kumar $\mathbf{V}^{1}$ \\ ${ }^{1}$ Department of cardiology, National Heart Institute, India \\ 2Department of cardiovascular surgery, National Heart Institute, India
}

\section{Case Report}

Volume 2 Issue 2

Received Date: May 09, 2018

Published Date: May 18, 2018

*Corresponding author: Hakim Irfan Showkat, DNB Cardiology Scholar, National Heart Institute, East of Kailash, New Delhi, India 110065, Tel: 09990390305/9419028326; Email: docirfanshahi512@gmail.com

\begin{abstract}
Mechanical valve dysfunction can be classified as endogenous and exogenous according to the etiology. Endogenous dysfunctions are caused by valve damage or defect, which has become extremely rare with the improvement of design, materials, and manufacture and detection methods in vitro. Exogenous causes include inappropriate selection of prosthesis, technical issues or other complications, such as thrombosis, excessive pannus overgrowth into the prosthetic rim, excessively long knot end, residual chordate tendineae stuck in the prosthetic sewing ring, extremely long residual papillary muscles in left ventricle or calcified tissues under the prosthesis hampering leaflet mobility. We are reporting a case of intermittent malfunction of prosthetic aortic valve [21 mm Medtronic hall mechanical prosthesis] in a 37-year-old female, who underwent Aortic Valve Replacement (AVR) eighteen year back for aortic valve endocarditis and severe Aortic Regurgitation (AR).
\end{abstract}

Keywords: Aortic; Prosthetic Valve; Dysfunction

Abbrevations: AVR: Aortic Valve Replacement; AR: Aortic Regurgitation; INR: International Normalized Ratio; TEE: Trans-Esophageal Echocardiography; TTE: Transthoracic Echocardiography; AVR: Aortic Valve Regurgitation; HF: Heart Failure.

\section{Case Report}

A Thirty seven year old woman underwent aortic valve replacement $[21 \mathrm{~mm}$ Medtronic hall mechanical prosthesis] for aortic valve endocarditis with severe aortic regurgitation in 1999. She was doing fairly well for these years and now this time she presented with intermittent episodes of chest discomfort, palpitation and breathlessness which used to subside after 10-15 minutes since last 5-6 days. She was admitted with initial normal ECG and 2Decho revealing high gradients across aortic valve with normal LV and prosthetic valve function. She became symptomatic while in hospital and ECG showed sinus tachycardia with ST depression in leads I, II, III, 


\section{Open Access Journal of Cardiology}

augmented vector left (a VF) and V3-V6.Acute Coronary Syndrome was suspected as she had raised cardiac enzymes, so immediate coronary angiography was done which revealed normal coronary arteries with normal prosthetic valve opening and closing on fluoroscopy. She was stabilized and further evaluation and management was planned. Her initial international normalized ratio (INR) was 1.675 and had audible valve click. The patient developed similar complaints while in ICU and repeat ECG showed sinus tachycardia with transient ST elevation in the leads augmented vector right (a VR) and V1 and ST depression in leads I, II, III, augmented vector left (a VF) and V3-V6 (Figure 1), but this resolved within ten to fifteen minutes. Auscultation at that time revealed the absence of a valve click and hypotension (systolic blood pressure of $80 \mathrm{~mm} \mathrm{hg}$ ). The patient recovered spontaneously, and a repeat ECG showed no significant abnormalities. Patient was planned for trans-esophageal echocardiography (TEE) but when she reached TEE room, she became symptomatic and transthoracic echocardiography (TTE) during episode revealed acute severe aortic regurgitation, moderate Mitral valve regurgitation with restricted prosthetic valve motion (Figure 2).

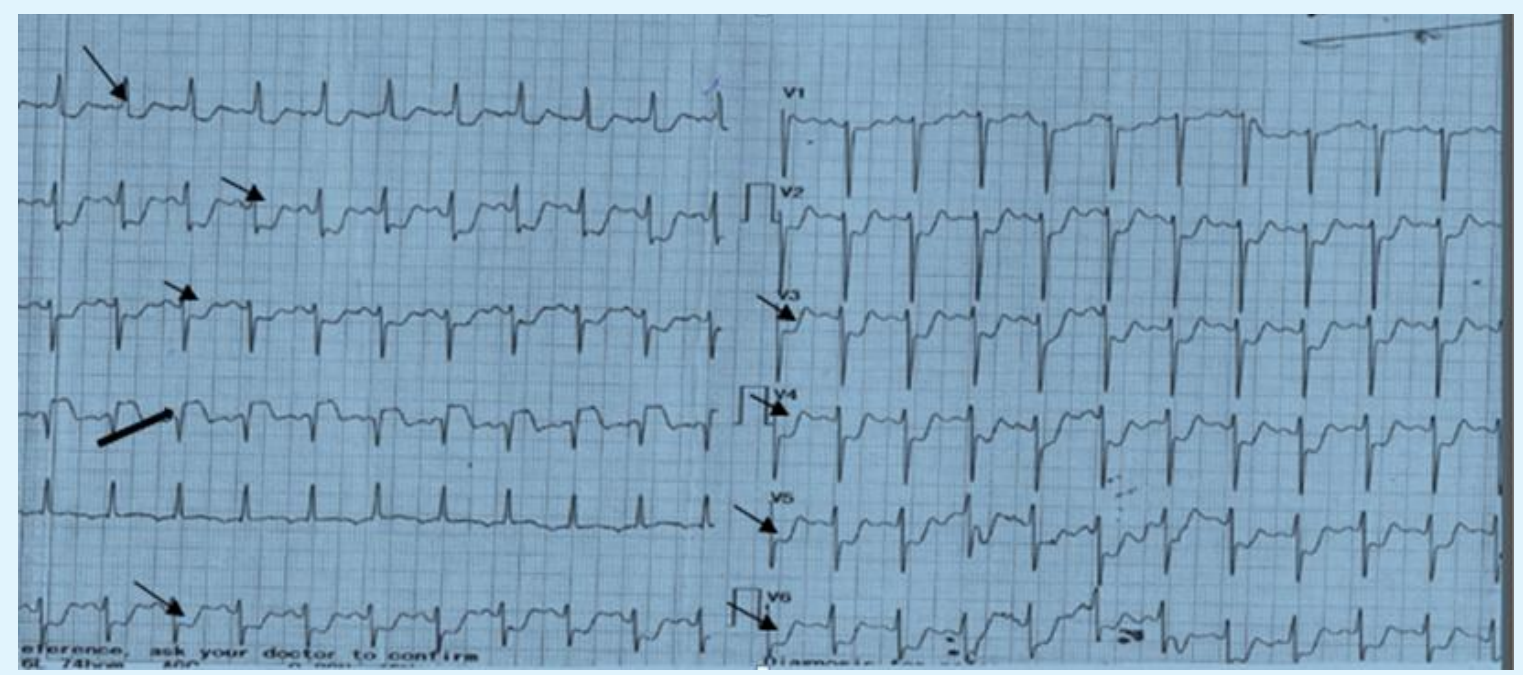

Figure 1: ECG showing sinus tachycardia with ST elevation in a VR (Big black arrow) and ST depression in leads I, II, III, a VF and V3-V6 (small black arrows).

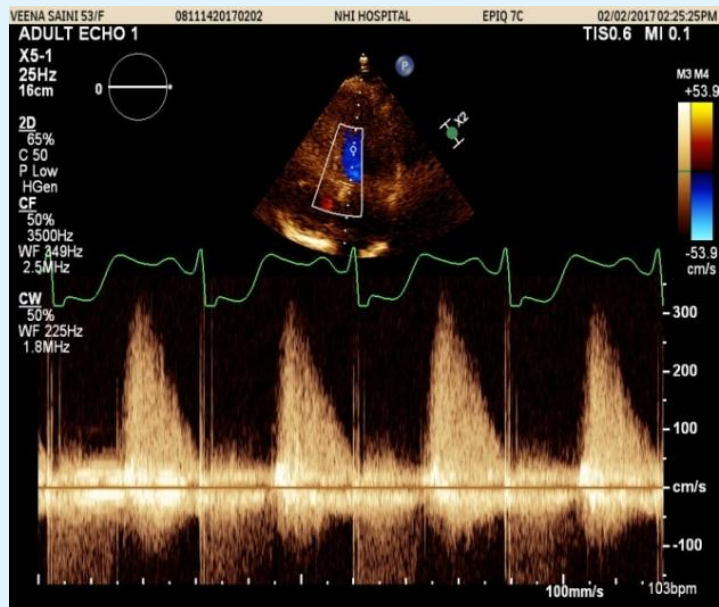

(a)

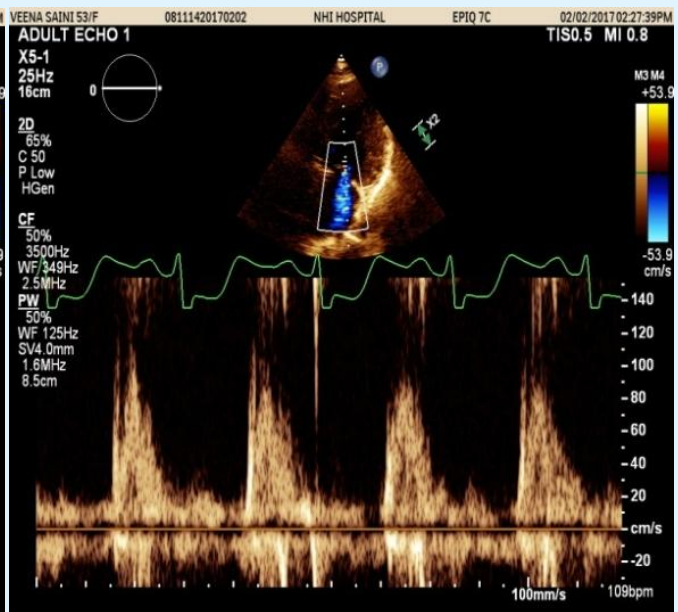

(b)

Figure 2a \& b: Echo/Doppler Showing Acute Severe AR \& mild-moderate MR. 


\section{Open Access Journal of Cardiology}

She was taken for surgical intervention. Pannus was found underneath the lesser orifice of the prosthetic valve (Figures 3a \& b). Aortic prostheses were explanted and new aortic valve was replaced using size $19 \mathrm{~mm}$ st Jude regent mechanical prosthesis. Postoperative echocardiography showed a normally functioning aortic valve, with normal gradients. Her postoperative course was uneventful, and the patient was discharged after 5 days. On follow-up, the patient is asymptomatic and normally functioning aortic prosthesis on 2D echocardiography.

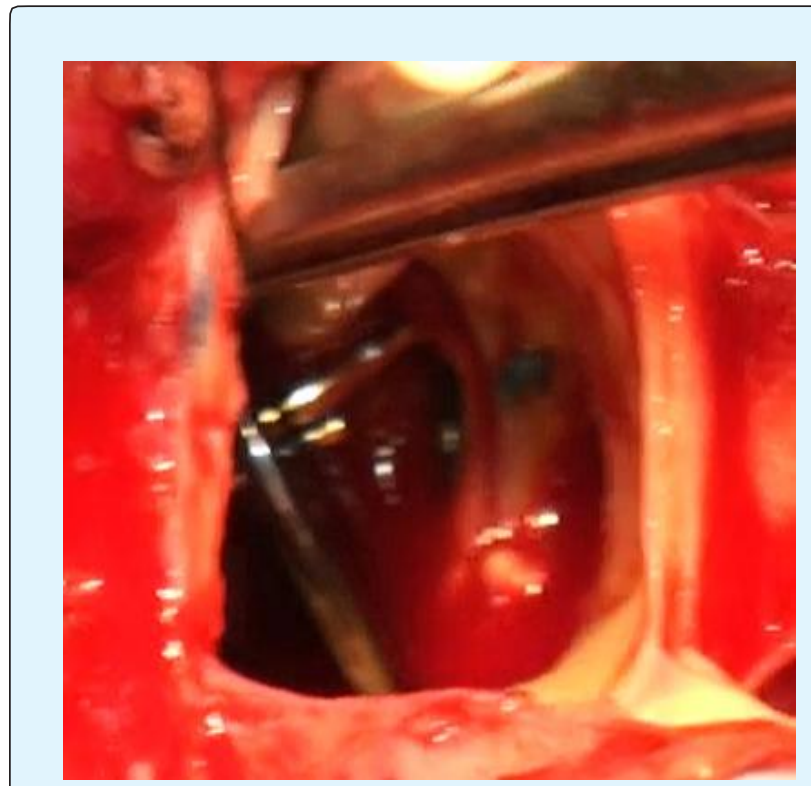

(a)

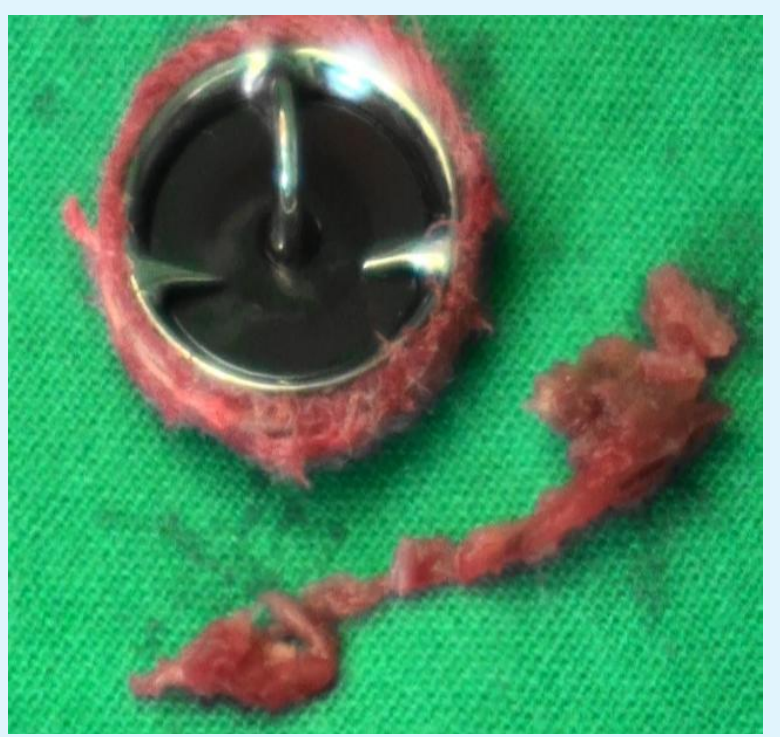

(b)

Figure 3a \& b: Valve in situ-opened showing Pannus underneath \& explanted valve with the surrounding rim of pannus.

\section{Discussion}

Mechanical valve dysfunction can be classified as endogenous and exogenous according to the etiology. Endogenous dysfunctions are caused by valve damage or defect, which has become extremely rare with the improvement of design, materials and manufacture and detection methods in vitro. Exogenous causes include inappropriate selection of prosthesis, technical issues or other complications, such as thrombosis, excessive pannus overgrowth into the prosthetic rim, excessively long knot end, residual chordate tendineae stuck in the prosthetic sewing ring, extremely long residual papillary muscles in left ventricle or calcified tissues under the prosthesis hampering leaflet mobility. The most common causes of mechanical valve dysfunction are thrombosis and pannus formation, which was present in $87.5 \%$ of patients in this series [1]. In comparison with the mitral position, intermittent prosthetic regurgitation in the aortic position is very rare. Prosthetic valve dysfunction as a result of pannus formation due to fibrous tissue ingrowths is an infrequent but a serious complication.
However, the mechanism of pannus formation has not been fully described yet and hence effective preventive methods have not been developed [2]. Usually, the pannus produces a stenos is of the prosthesis due to obstruction of the LV outflow tract or restriction on the movement of the opening of the discs of the prosthetic valve [3]. In our patient AR was due to pannus underneath the lesser orifice of prosthetic valve. This impeded the normal closing of the leaflet intermittently, leading to the phasic AR. Clinical signs and auscultation may be confusing and unhelpful in the evaluation of the severity of valvular dysfunction. Murmurs may be heard in normally functioning valves whereas Para valvular regurgitation may be silent Single tilting disc prostheses are more prone to develop this condition, and it has also been linked tosubvalvular no obstructing pannus [2,4]. In addition, intermittent prosthetic aortic valve regurgitation (AVR) due to malfunction can present as severe acute ischemia from the load/perfusion mismatch or as heart failure (HF) as in our case. Given the fact that the valve may appear almost normal in between the episodes, valve malfunction may be difficult to identify 


\section{Open Access Journal of Cardiology}

$[5,6]$. The absence of an audible click, repeat transthoracic echocardiographic interrogation of the valve during the episode of hemodynamic instability will help to make correct diagnosis. And obviously management will be surgical as per the correct diagnosis.

\section{Conclusion}

An intermittent noncyclical dysfunction may not be obvious at the time of clinical examination. So under these conditions and as TEE cannot be repeated promptly every time, transthoracic 2-D and Doppler echocardiography should be available at any time when symptoms develop. As this may be life threatening condition TTE is the method of choice for acute patient evaluation and urgent referral for surgical management is a rule.

\section{References}

1. Ma WG, Hou B, Abdurusul A, Gong DX, Tang Y, et al. (2015) Dysfunction of mechanical heart valve prosthesis: experience with surgical management in 48 patients. Journal of Thoracic Disease 7(12): 23212329.

2. Aoyagi S, Nishimi M, Tayama E, Fukunaga S, Hayashida N, et al. (2002) Obstruction of St Jude medical valves in the aortic position: a consideration for pathogenic mechanism of prosthetic valve obstruction. Cardiovasc Surg 10(4): 339-344.

3. Cianciulli TF, Saccheri MC, Lax JA, Guidoin R, Zhang Z, et al. (2009) Intermittent acute aortic regurgitation of a mechanical bileaflet aortic valve prosthesis: diagnosis and clinical implications. European Journal of Echocardiography 10(3): 446-449.

4. Lengyel M, Vandor L (2001) The role of thrombolysis in the management of leftsided prosthetic valve thrombosis: study of 85 cases diagnosed by transesophageal echocardiography. J Heart Valve Dis 10(5): 636-649.

5. Karagiannis SE, Karatasakis G, Spargias K, Louka L, Poldermans D, et al. (2008) Intermittent acute aortic valve regurgitation: a case report of a prosthetic valve dysfunction. Eur J Echocardiogr 9(2): 291-293.

6. Giroux SK, Labinaz MX, Grisoli D, Klug AP, Veinot JP, et al. (2010) Intermittent, noncyclic dysfunction of a mechanical aortic prosthesis by pannus formation. J Am Soc Echocardiogr 23(1): 107.e1-3. 